\title{
Are Solifenacin and Ramosetron Really Ideal to Treat Irritable Bowel Syndrome?: Author's Reply
}

TO THE EDITOR: We appreciate the thoughtful comments from Chang ${ }^{1}$ in regards to our recent paper. ${ }^{2}$ The overall improvement was assessed on the basis of the patients' subjective reports during their interview regarding whether the symptoms had improved, compared to before administering solifenacin. Since the overall improvement is an established index often used in determining the therapeutic efficacy of irritable bowel syndrome (IBS), we do not think that the extremely high efficacy of solifenacin in our paper ${ }^{2}$ have been caused by the setting of overall improvement as a primary endpoint. Differences between the enrolled populations could be a possible cause. Most of the previous studies on IBS have been "tertiary-care-hospital-based," however, this study was "health clinic-based." Most of the patients had not received any treatment, despite the presence of IBS symptoms. In addition, because no placebo group was established and participants were aware that the medication, they were taking, was the actual drug, the placebo effect might be stronger than previously reported. For this reason, the actual therapeutic efficacy of solifenacin in the treatment of IBS will have to be verified using a placebo-controlled study.

As Chang ${ }^{1}$ mentioned, setting up a washout period and evaluating IBS symptoms before and after administration of ramosetron has allowed more accurate understanding of the effects of ramosetron. Meanwhile, solifenacin has a half-life of 45 to 68 hours. ${ }^{3}$ Because the score after the 4-week administration of ramosetron reflected the symptoms from the third week to the fourth week after administration of ramosetron, it can be considered that the score is unlikely to be affected by the residual pharmacological effect of solifenacin.

Since Figure 2A to 2F (including 2C) demonstrate the aver- age scores of the subscales of IBS-symptom severity scale, the y-axis was labeled as "scores."

As constipation has been pointed out as a side effect of solifenacin, precaution for constipation must be taken when using solifenacin in the medical treatment of overactive bladder. ${ }^{4}$ Although the mechanism that solifenacin was effective for the treatment of diarrhea predominant IBS could not be elucidated, it would be reasonable to believe that the improvement of diarrhea might have been attributable to the same mechanism as that by which solifenacin caused constipation. In the future, when using solifenacin in the treatment of diarrhea predominant IBS, it would be appropriate to conduct the same dose adjustment as we performed, after administering the dose for 1 to 2 weeks.

Hidekazu Suzuki and Juntaro Matsuzaki

Department of Internal Medicine, Keio University School of Medicine Tokyo, Japan

1. Chang FY. Are solifenacin and ramosetron really ideal to treat irritable bowel syndrome? J Neurogastroenterol Motil 2012;18:457-458.

2. Fukushima Y, Suzuki H, Matsuzaki J, Kiyosue A, Hibi T. Efficacy of solifenacin on irritable bowel syndrome with diarrhea: open-label prospective pilot trial. J Neurogastroenterol Motil 2012;18:317-323.

3. Basra R, Kelleher C. A review of solifenacin in the treatment of urinary incontinence. Ther Clin Risk Manag 2008;4:117-128.

4. Matsuzaki J, Suzuki H, Fukushima Y, et al. High frequency of overlap between functional dyspepsia and overactive bladder. Neurogastroenterol Motil 2012;24:821-827.

\section{Conflicts of interest: None.}

